\title{
Novel Hyaluronate Lyase Involved in Pathogenicity of Streptococcus dysgalactiae subsp. equisimilis
}

\author{
Van An Nguyen ${ }^{1}$, Kohei Ogura ${ }^{2 *}$, Miki Matsue ${ }^{3}$, Norihiko Takemoto ${ }^{4}$, Kanae Mukai ${ }^{5}$, \\ Yukari Nakajima ${ }^{5}$, Thuy Linh Hoang ${ }^{6}$, Yasunori Iwata ${ }^{6,7}$, Norihiko Sakai ${ }^{6}$, Takashi Wada ${ }^{6,8}$, \\ Wataru Hashimoto ${ }^{9}$, Shigefumi Okamoto ${ }^{3}$ and Hiroshi Ichimura ${ }^{1}$
}

\begin{abstract}
${ }^{1}$ Department of Viral Infection and International Health, Graduate School of Medical Sciences, Kanazawa University, Kanazawa, Japan, ${ }^{2}$ Advanced Health Care Science Research Unit, Institute for Frontier Science Initiative, Kanazawa University, Kanazawa, Japan, ${ }^{3}$ Department of Clinical Laboratory Science, Faculty of Health Sciences, Institute of Medical, Pharmaceutical and Health Sciences, Kanazawa University, Kanazawa, Japan, ${ }^{4}$ Pathogenic Microbe Laboratory, Research Institute, National Center for Global Health and Medicine, Shinjuku, Japan, ${ }^{5}$ Department of Nursing Sciences, Faculty of Health Sciences, Institute of Medical, Pharmaceutical and Health Sciences, Kanazawa University, Kanazawa, Japan, ${ }^{6}$ Department of Nephrology and Laboratory Medicine, Kanazawa University, Kanazawa, Japan, ${ }^{7}$ Division of Infection Control, Kanazawa University, Kanazawa, Japan, ${ }^{8}$ Department of Clinical Laboratory, Kanazawa University Hospital, Kanazawa, Japan, 'ªboratory of Basic and Applied Molecular Biotechnology, Division of Food Science and Biotechnology, Graduate School of Agriculture, Kyoto University, Uji, Japan
\end{abstract}

Streptococcus dysgalactiae subsp. equisimilis (SDSE) causes cellulitis, bacteremia, and invasive diseases, such as streptococcal toxic shock syndrome. Although SDSE infection is more prevalent among elderly individuals and those with diabetes mellitus than infections with Streptococcus pyogenes (Group A streptococci; GAS) and Streptococcus agalactiae (Group B streptococci; GBS), the mechanisms underlying the pathogenicity of SDSE remain unknown. SDSE possesses a gene hylD encoding a hyaluronate lyase (HyID), whose homologue (HylB) is involved in pathogenicity of GBS, while the role of HyID has not been characterized. In this study, we focused on the enzyme HyID produced by SDSE; HyID cleaves hyaluronate $(\mathrm{HA})$ and generates unsaturated disaccharides via a $\beta$-elimination reaction. Hyaluronate-agar plate assays revealed that SDSE promoted dramatic HA degradation. SDSE expresses both HyID and an unsaturated glucuronyl hydrolase (UGL) that catalyzes the degradation of HA-derived oligosaccharides; as such, SDSE was more effective at HA degradation than other $\beta$-hemolytic streptococci, including GAS and GBS. Although HylD shows some homology to HylB, a similar enzyme produced by GBS, HylD exhibited significantly higher enzymatic activity than $\mathrm{HylB}$ at $\mathrm{pH}$ 6.0, conditions that are detected in the skin of both elderly individuals and those with diabetes mellitus. We also detected upregulation of transcripts from hyID and ugl genes from SDSE wild-type collected from the mouse peritoneal cavity; upregulated expression of ug/ was not observed in $\triangle$ hyID SDSE mutants. These results suggested that disaccharides produced by the actions of HylD are capable of triggering downstream pathways that catalyze their destruction. Furthermore, we determined that infection with SDSE $\triangle$ hylD was significantly less lethal than infection with the parent strain. When mouse skin wounds were infected for 2 days, intensive infiltration of neutrophils was observed around the wound areas 
infected with SDSE wild-type but not SDSE $\triangle$ hyID. Our investigation suggested that HyID and UGL play important roles in nutrient acquisition from hosts, followed by the bacterial pathogenicity damaging host tissues.

Keywords: Streptococcus dysgalactiae subsp. equisimilis, hyaluronate, hyaluronate lyase, pathogenicity, Streptococcus agalaciate, skin pH

\section{INTRODUCTION}

Streptococcus pyogenes (Group A streptococci; GAS), Streptococcus agalactiae (Group B streptococci; GBS), and Streptococcus dysgalactiae subsp. equisimilis (SDSE) are $\beta$-hemolytic streptococci. Takahashi et al. (2011) reported that SDSE had Lancefield group $\mathrm{G}$ or $\mathrm{C}$ antigens on their cell surface, whereas our group recently reported on the prevalence of Lancefield group A SDSE in Japan (Ishihara et al., 2020). SDSE colonizes the pharynx, gastrointestinal tract, female genital tract, and skin as a commensal microorganism (Yung et al., 2019) and is involved in a wide variety of infectious diseases from relatively minor wound infections, erysipelas, and cellulitis to life-threatening necrotizing fasciitis and streptococcal toxic shock syndrome (STSS; Brandt and Spellerberg, 2009; Rantala, 2014). Genomic analysis revealed that several virulence factors are conserved in both GAS and SDSE (Shimomura et al., 2011). However, SDSE lacks several virulence factors, including the streptococcal pyrogenic exotoxin (spe) and hyaluronate (HA) synthesis (has A and hasB) genes. GAS and GBS produce and secrete HA that forms a bacterial capsule that plays a critical role in promoting immune evasion and pathogenicity (Stollerman and Dale, 2008). Transcription of the GAS HA biosynthesis operon is regulated by a two-component CovR-CovS (also known as CsrR-CsrS) regulatory system; loss of CovR-CovS function results in overexpression of HA (Heath et al., 1999; Falaleeva et al., 2014). SDSE does not produce HA, and as such, deletion of this regulatory system from these bacteria does not result in the generation of mucoid colonies (Watanabe et al., 2013). Of interest, we recently reported that SDSE utilizes cell wall-anchoring proteins as envelopes in serum-containing medium (Matsue et al., 2020).

In the human tissues, $\mathrm{HA}$ is a critical component of the extracellular matrix and functions by retaining water molecules (Baumann, 2007). Approximately 50\% of human HA is associated with the integumentary system (Reed et al., 1988; Juhlin, 1997) where it functions to create a semi-permeable barrier. Some of streptococcal strains are capable of degrading HA via the production of hyaluronate lyases (Hyls; ENZYME entry: EC 4.2.2.1); Hyls are enzymes that cleave $\beta 1,4$ linkages in HA via a $\beta$-elimination reaction (Hynes, 2004; Figure 1A). Unsaturated HA oligosaccharides produced by the actions of Hyls are further degraded by unsaturated glucuronyl hydrolase (UGL), an enzyme that catalyzes the hydrolysis of oligosaccharides with unsaturated glucuronyl residues at their non-reducing termini (Hashimoto et al., 1999; Maruyama et al., 2009). hylP genes encoding active Hyls in GAS strains were detected in specific bacteriophages, whereas clinical isolates of SDSE encode hylD genes that are homologous to $h y l B$ genes from S. agalactiae (Shimomura et al., 2011). Hyaluronate lyase
A

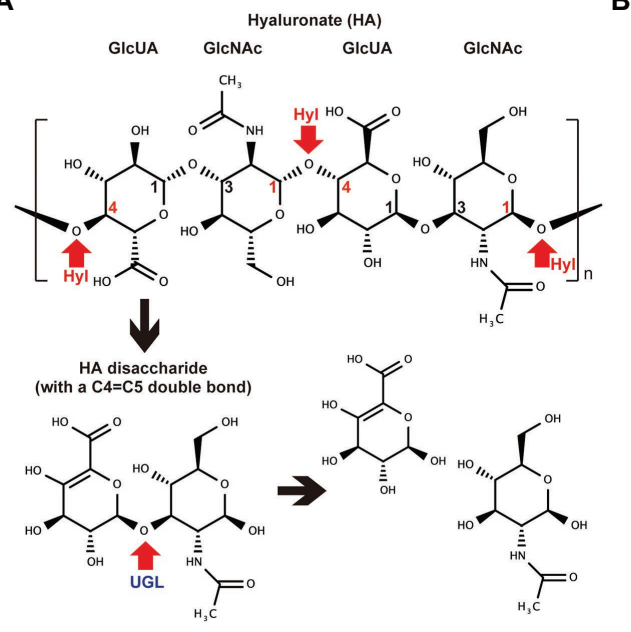

B

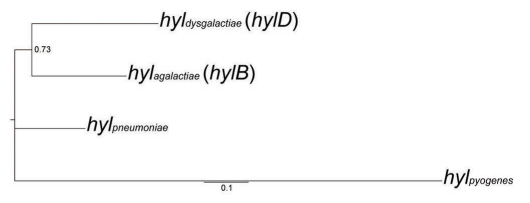

C

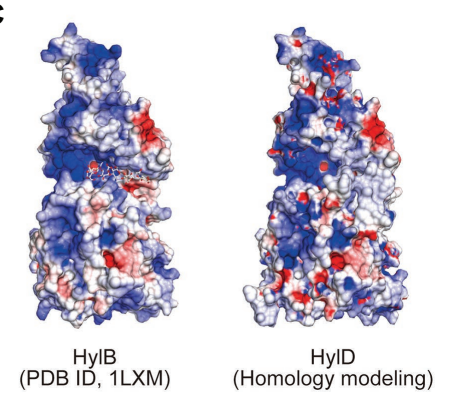

FIGURE 1 | Streptococcal hyaluronate lyases. (A) Schematic diagram of hyaluronate (HA) degradation of Hyl and UGL. $\beta 1,4$ linkages between glucuronic acid (GlcUA) and N-acetylglucosamine (GlcNAc) were degraded by hyaluronate lyase (Hyl; represented in red arrows). The resultant unsaturated HA disaccharides were hydrolyzed by unsaturated glucuronyl hydrolase (UGL; represented by a red arrow). The chemical formula was drawn by MarvinSketch software (ChemAxon). (B) Genetic diversity of streptococcal hyaluronate lyases (Hyls) from S. pyogenes strain 476 (locus tag: M1GAS476_0819), Streptococcus pneumoniae strain R6CIB17 (E5Q10_01570), Streptococcus agalactiae strain COH1 (GBSCOH1_1103), and Streptococcus dysgalactiae subsp. equisimilis (SDSE) strain 124 (SDEG_0654). (C) Structural similarity of HyIB (PDB ID, 1LXM) and HyID (homology modeling using 1LXM) from S. agalactiae and SDSE, respectively. 
from GBS (HylB) functions as a virulence factor in the human tissues. Interestingly, HylB-derived HA disaccharides resulted in reduced levels of pro-inflammatory cytokine when administered as treatment in a mouse acute lung injury model; these results suggest that GBS can degrade host-generated pro-inflammatory HA fragments via the actions of HylB and thereby may escape from host immune-mediated detection (Kolar et al., 2015).

Streptococcus dysgalactiae subsp. equisimilis has been identified as the etiologic agent in severe invasive streptococcal infections in the elderly (Wajima et al., 2016); it is unclear what promotes this apparent high pathogenicity compared with that seen in infections with GAS and GBS. GAS and GBS both produce and degrade HA, whereas SDSE is capable of HA degradation only; these results suggest that SDSE-mediated regulation of $h y l$ and its downstream factors proceed differently from that identified in GAS and GBS. Furthermore, aging changes the characteristics of the skin; among the best characteristics of these changes are the reduced production of sebum and higher pH (Choi et al., 2007; Levin and Maibach, 2008). The skin $\mathrm{pH}$ of patients with diabetes mellitus is also higher than that detected in healthy subjects (Behm et al., 2012). Interestingly, Meyrer et al. reported that HA concentration and polymer size of HA in the human skin do not undergo change as a function of age (Meyer and Stern, 1994). On the basis of these characteristics, we hypothesized that the pathogenicity of SDSE might relate to its capacity to degrade HA. In this study, we focused on the role of HylD, the hyaluronate lyase from SDSE, and explored its role in promoting pathogenicity. Toward this end, we examined the expression of hylD in bacteria grown in culture medium and in the intraperitoneal cavity of the infected mice. We measured the $\mathrm{pH}$-dependent enzymatic activity of HylD and HylB and examined the impact of hylD gene deletion on SDSE-mediated lethality in infected mice.

\section{MATERIALS AND METHODS}

\section{Phylogenetic Analysis}

Phylogenetic analysis was conducted using MEGA 5 software with neighbor joining method (Tamura et al., 2011) for streptococcal Hyls from S. pyogenes strain 476 (locus tag: M1GAS476_0819), Streptococcus pneumoniae strain R6CIB17 (E5Q10_01570), S. agalactiae strain COH1 (GBSCOH1_1103), and SDSE strain 124 (SDEG_0654). Tree was visualized by Fig Tree. ${ }^{1}$

\section{Homology Modeling}

The protein structure of HylD was predicted by UCSF Chimera and MODELER using HylB (PDB ID, 1LXM) as a template (Pettersen et al., 2004; Eswar et al., 2006). Protein surface and electric charge were visualized by CueMol software (Molecular Visualization Framework; http://www.cuemol.org/). Isoelectric points ( $\mathrm{pIs}$ ) were computed based on their primary protein structure (amino acid sequences) using the ExPASy website (Bjellqvist et al., 1993).

${ }^{1}$ http://tree.bio.ed.ac.uk/software/figtree/

\section{Bacterial Strains}

Bacterial strains SDSE-124 (GenBank accession number AP010935.1), SDSE-C167 (AP012976.1), GAS M1 476 (AP012491.2), GBS COH1 (HG939456.1), and GBS A909 (CP000114.1) were utilized in this study. Additional bacterial strains included TPCH-F36, which was isolated in Toyama Prefecture Central Hospital (Japan); KNZ01, KNZ03, KNZ10, and KNZ16 from Kanazawa University Hospital, Japan (Matsue et al., 2020); and GAS M1 SMD from National Center of Global Health and Medicine (Japan). The bacteria utilized in this study were cultured in Todd Hewitt broth (Becton Dickinson, Franklin Lakes, NJ, USA) supplemented with $0.2 \%$ yeast extract (THY, Becton Dickinson) or brain-heart infusion (BHI, Becton Dickinson); bacterial cultures were grown overnight at $37^{\circ} \mathrm{C}$ in a $5 \% \mathrm{CO}_{2}$ atmosphere. All experiments were conducted in accordance with the WHO Laboratory Biosafety Manual and institutional safety procedures at Kanazawa University.

\section{Preparation of the $\Delta$ hylD Mutant Strain}

hylD gene in SDSE-124 (locus tag: SDEG_0654) was deleted using thermosensitive suicide vector pSET4S according to a previous study (Takamatsu et al., 2001; Supplementary Figure S1). One kilo byte upstream $(620,723-621,724)$ and $1 \mathrm{~kb}$ downstream $(624,929-625,928)$ sequence of the $h y l D$ gene were concatenated by overlap extension PCR using Gflex DNA polymerase (Takara Bio, Japan) and cloned into EcoRI-digested pSET4S using an In-Fusion HD Cloning Kit (Takara Bio). SDSE-124 was transformed by electroporation with the constructed plasmid. After incubation at $28^{\circ} \mathrm{C}$ for $4 \mathrm{~h}$, the cells were spread on a $250-\mu \mathrm{g} / \mathrm{ml}$ spectinomycin-containing BHI (Sp250-BHI) plate, incubated at $28^{\circ} \mathrm{C}$ for 2 days, and collected in Sp250-BHI medium. Single-crossover spectinomycin-resistant mutant cells were obtained after incubation in Sp250-BHI medium and spreading on Sp250-BHI plates at $28^{\circ} \mathrm{C}$. The single-crossover mutants were repeatedly incubated at $28^{\circ} \mathrm{C}$ in $\mathrm{BHI}$ (no spectinomycin). The spectinomycin-sensitive colonies were considered to be double-crossover strains with $\Delta$ hylD. The single- and double-crossovers were confirmed by colony direct PCR using Gflex DNA polymerase. The resultant sequences were confirmed using an Applied Biosystems 3130xl Genetic Analyzer.

\section{Hyaluronate Degradation Halo Assay}

Degraded HA halo assays were performed as previously described with some modifications (Smith and Willett, 1968; Oiki et al., 2019). Agar plates consisting of $0.5 \%$ peptone (Becton Dickinson, Franklin Lakes, NJ, USA), 0.3\% beef extract (Becton Dickinson, Franklin Lakes, NJ, USA), 1\% agar, 1\% bovine serum albumin (Nacalai Tesque, Japan), and $0.4 \mathrm{mg} / \mathrm{ml}$ hyaluronic acid (FUJIFILM Wako Pure Chemical Corporation, Japan) were prepared. Bacterial strains were grown in BHI medium, washed with fresh $\mathrm{BHI}$, and resuspended in fresh $\mathrm{BHI}$ at the indicated optical density at $600 \mathrm{~nm}\left(\mathrm{OD}_{600}\right)$. One microliter of bacterial suspensions at the indicated concentration was spotted on the peptone agar, followed by overnight incubation at $37^{\circ} \mathrm{C}$ in a $5 \% \mathrm{CO}_{2}$ atmosphere. The plates were then flooded with $2 \mathrm{~N}$ acetic acid and allowed to stand for $10 \mathrm{~min}$. Clear regions demarcate zones of HA degradation. 


\section{Quantitative Real-Time PCR}

Bacteria were collected in RNAprotect Bacteria Reagent (Qiagen, Hilden, Germany). After treatment with $20 \mathrm{mg} / \mathrm{ml}$ lysozyme (FUJIFILM Wako Pure Chemical Corporation) and $50 \mathrm{U} / \mathrm{ml}$ mutanolysin (Merck) at $37^{\circ} \mathrm{C}$ for $90 \mathrm{~min}$, bacterial RNAs were extracted with NucleoSpin RNA kit (Macherey-Nagel, Düren, Germany), treated with gDNA Eraser (Takara Bio, Japan), and reverse-transcribed with PrimeScript RT reagent Kit according to the manufacturers' protocols. Quantitative real-time PCR (RT-qPCR) was conducted in AriaMx Real-Time PCR System (Agilent Technologies) using a QuantiFast SYBR Green PCR Kit (Qiagen) or a QuantiTect Probe PCR Kit (Qiagen). Primers and probes for quantification of $h y l, u g l$, and mutS expression are listed in Table 1. The oligonucleotides were purchased from Eurofin Genomics, Japan. Standard curves for quantitative analysis of $h y l$, ugl, and mutS gene expression were prepared with the indicated copy numbers of genes inserted into plasmids PQE30/ hylB, pQE30/hylD, pQE30/GBS_ugl, and pQE30/SDSE_ugl.

\section{Growth Assays}

The chemically defined medium (CDM) was prepared as previously described (van de Rijn and Kessler, 1980) and supplemented with HA $(0.4 \mathrm{mg} / \mathrm{ml}), \mathrm{HA}(2.0 \mathrm{mg} / \mathrm{ml})$, or glucose $(10 \mathrm{mg} / \mathrm{ml})$. The amounts of carbon sources were chosen according to HA concentration in human skin (normally approximately $0.4 \mathrm{mg} /$ $\mathrm{ml})$, and knee joint HA $(2.0 \mathrm{mg} / \mathrm{ml})$ and fasting blood glucose (10 mg/ml) concentrations (Balazs et al., 1967; Dahl et al., 1985; Tammi et al., 1994). All media were filtered through a $0.45 \mu \mathrm{m}$-poresize filter prior to use. SDSE-124, SDSE-124 $\Delta h y l D$, GBS-COH1, and GAS-476 were grown in THY medium, washed with fresh CDM with no added carbohydrate [CDM (-)], and resuspended in fresh CDM (-). A specific amount of bacterial suspension was transferred into $3 \mathrm{ml}$ of CDM with or without added carbohydrate to achieve an optical density of 0.05 at $600 \mathrm{~nm}$. These bacterial suspensions were incubated at $37^{\circ} \mathrm{C}$ in a $5 \% \mathrm{CO}_{2}$ atmosphere. $\mathrm{OD}_{600}$ was measured with a Genesys 20 spectrophotometer (Thermo Scientific, USA) and the bacterial growth in each medium was assessed in three independent tests, in triplicate.

\section{Overexpression of $\mathrm{Hyl}$ and UGL}

Hyaluronate lyases from GBS-COH1 and SDSE-124 were overexpressed in Escherichia coli. We could not obtain full-length Hyl. Since a previous report showed that Hyl undergoes an autocatalytic conversion to a smaller enzymatically active form (Jedrzejas and Chantalat, 2000), we utilized truncated versions of HylB and HylD, both with N-terminal 170 amino acid deletions. The $h y l$ and $u g l$ genes were cloned into pCold I (Takara Bio) using a Gibson Assembly Kit (New England Biolabs, USA). BL21(DE3) was transformed with the resultant plasmid, cultivated, and homogenized for recombinant protein purification with TALON Metal Affinity Resin (Takara Bio). After buffer exchange with $5 \mathrm{mM}$ HEPES- $\mathrm{NaOH}$ (pH 7.0) using PD-10 columns (GE Healthcare, USA), the purified recombinant protein solutions were utilized for enzymatic assays. Protein concentrations were measured by BCA Protein Assay kit (ThermoFisher Scientific).

\section{Enzymatic Activity of HylB and HyID}

Ten microliters of the enzyme (recombinant HylB or HylD) solution $(0.05 \mathrm{mg} / \mathrm{ml})$ were mixed with $500 \mu \mathrm{l}$ of $1 \mathrm{mg} / \mathrm{ml} \mathrm{HA}$ solution containing $0.2 \mathrm{M}$ buffer (described below) and left at $37^{\circ} \mathrm{C}$. The increase in absorbance at $235 \mathrm{~nm}$ due to $\mathrm{C}=\mathrm{C}$ double bond formation was monitored with a U-3210 spectrometer (Hitachi Industries, Japan) at a final pH of 5.0 (in the presence of citrate buffer), 5.5 (citrate), 6.0 (MOPS- $\mathrm{NaOH}$ ), 6.5 (MOPS$\mathrm{NaOH}$ ), 7.0 (MOPS- $\mathrm{NaOH}$ ), 7.5 (HEPES- $\mathrm{NaOH}$ ), 8.0 (HEPES$\mathrm{NaOH}$ ), 8.5 (glycine- $\mathrm{NaOH}$ ), or 9.0 (glycine- $\mathrm{NaOH}$ ).

\section{Product Analysis With Thin Layer Chromatography}

Five microliters of recombinant HylD and/or UGL solution (0.1 mg/ ml) was mixed with $100 \mu \mathrm{l}$ of $1 \mathrm{mg} / \mathrm{ml} \mathrm{HA}$ solution containing $0.1 \mathrm{M}$ citrate buffer $\mathrm{pH} 6.0$ and left overnight at $37^{\circ} \mathrm{C}$. Ten microliters of the solutions were spotted on a silicagel 70 TLC plate (FUJIFILM Wako Pure Chemical Corporation). The resulting products were separated with thin layer chromatography using a 1-butanol/acetic acid/water (4:1:1, v/v) solvent system. The products were visualized after spraying them with $5 \%$ sodium phosphomolybdate n-hydrate (FUJIFILM Wako Pure Chemical Corporation) in ethanol and heating to $200^{\circ} \mathrm{C}$ for $5 \mathrm{~min}$.

\section{Mouse Intraperitoneal Injection Assays}

Streptococcus dysgalactiae subsp. equisimilis strains were cultured in BHI. Bacterial cells were harvested by centrifugation and washed with ice-cold fresh $\mathrm{BHI}$ when the culture was in its exponential phase $\left(\mathrm{OD}_{600}=0.5\right)$. A $0.5-\mathrm{ml}$ aliquot containing approximately $2.0 \times 10^{6}$ colony-forming units (CFU) of SDSE-124

TABLE 1 | Primers and probes used in this study.

\begin{tabular}{ll}
\hline Bacteria/Genes & Forward and reverse primer sequences $\left.\mathbf{( 5}^{\prime} \mathbf{>} \mathbf{3}^{\prime}\right)$ \\
\hline For SYBR Green qPCR (Figure $\mathbf{2 C}$ ) & CCATTGATCAGCGAAAGGAT, AGACCAGCGTCCTGACTGT \\
SDSE-124/hyl & AATCCTTAATCAAGGCTCTTAGCAT, GGATTAATITTGGAGATGGAAGT \\
SDSE-124/ugl & GAGTCTGGCTTGGCTTCATC, CACCAAGGGCTTAAATGGA \\
GBS-COH1/hyl & GTGTCACACGACAGGGTTATAGTG, ATGTATCTCTAGGCTGACCCGA \\
GBS-COH1/ugl & CCAGATGCTTTTTGCTTTTAG, ACCTCACGCTTGACCAC \\
SDSE-124\&GBS-COH1/mutS & \\
For Probe qPCR (Figure 5A) & \\
SDSE-124/hyl & CGACTTCAACAGACAAAG, CAGGATTAATGACAACCG \\
SDSE-124/ugl & GGCATTCCTTGACCTATC, TCGTGGATACCACATACG \\
SDSE-124/mutS & CTTGCAATACGTTCACAA, CTAGCATTCTCTACCAAATC
\end{tabular}


(WT or $\Delta h y l D$ ) was used for intraperitoneal injection and the bacteria were recovered via injection of $5 \mathrm{ml}$ of fresh BHI at the indicated time points. To determine survival rates, 5 week-old C57BL/6 J mice (Sankyo Laboratory Service Corp., Inc., Tokyo, Japan) were injected intraperitoneally with $0.5 \mathrm{ml}$ containing approximately $2.0 \times 10^{6} \mathrm{CFU}$ of SDSE strains and observed once a day for 7 days according to a previous study (Watanabe et al., 2013). The volume for administration was determined according to a guideline (Diehl et al., 2001).

\section{Murine Wound Model}

Skin wounds were made according to a previous report with some modifications (Mukai et al., 2016), 5 week-old C57BL/6JJ mice were anesthetized through intraperitoneal administration of a mixture of Dormicum (4 mg/kg; Astellas Pharma, Inc., Tokyo, Japan), Vetorphale (5 mg/kg; Meiji Seika Pharma Co., Ltd., Tokyo, Japan), and Domitor (0.3 mg/kg; Nippon Zenyaku Kogyo Co., Ltd., Fukushima, Japan). The backs of the mice were shaved, and their skin was disinfected with ethanol. A circular full-thickness wound ( $4 \mathrm{~mm}$ in diameter) including the panniculus carnosus muscle was made using a sterile biopsy punch (Kai Industries Co. Ltd., Gifu, Japan). The wounds were covered with a transparent dressing (Tegaderm; 3 M Health Care, Tokyo, Japan) for $24 \mathrm{~h}$ to maintain a moist environment. Then, a $0.1-\mathrm{ml}$ aliquot containing approximately $1.4 \times 10^{6}$ CFUs of SDSE-124 (WT or $\Delta$ hylD) in PBS was inoculated directly onto the wounds of the mice under anesthesia, which were then covered with sterile gauze and transparent dressing. On day 2 post-infection, the infected mice were anesthetized with Sevofrane (Maruishi Pharmaceutical Co., Ltd., Osaka, Japan); then, blood was immediately collected for determination of Interleukin 6 levels (R\&D Systems, Minneapolis, $\mathrm{MN}$, USA), and tissue was excised from the skin lesion, fixed in $4 \%$ paraformaldehyde in PBS, and then embedded in paraffin. The tissue samples were stained with hematoxylin and eosin (H\&E; Muto Pure Chemicals Co., Ltd., Tokyo, Japan) and Gram stain (Nissui Pharma Medical Sales Co., Ltd., Japan) according to the manufacturer's instructions. An optical microscope (Eclipse E600 with U-III Film Camera System; Nikon Corp., Tokyo, Japan) was used to examine the stained tissue sections and images were captured using the NIS-Elements imaging software (Nikon).

\section{Ethics Statement}

All animal experiments were conducted in accordance with the Declaration of Helsinki and Act on Welfare and Management of Animal (Ministry of Environment, Japan). All genetic recombination experiments were approved by the Committee for Genetic Recombination Experiments (Kanazawa University).

\section{RESULTS}

\section{Genetic and Structural Characteristics of SDSE Hyaluronate Lyase}

As shown in the phylogenetic tree (Figure 1B), SDSE hyaluronate lyase gene (hylD, hyldysgalactiae) is closely related to the hylB gene of GBS hyl (hylB, hylagalactiae) and more distant from those encoded in the genomes of GAS (hylpyogenes) or
S. pneumoniae hyl (hylpneumoniae). Since the protein structure of SDSE Hyl (HylD) has not been determined, we generated a homology modeling prediction on the basis of the known structure of GBS Hyl (HylB), which has a $44 \%$ amino acid sequence identity to HylD. Although the computed pI of HylD was 5.4 and thus substantially different from that calculated for HylB ( $\mathrm{pI}=8.8)$, the predicted charges on the HylD protein surface were similar to those shown for HylB (Figure 1C). Interestingly, there were significant differences in the loop region $\left({ }_{804} \mathrm{KKLTID}_{809}\right)$, which includes the HA binding cleft (Supplementary Figure S2).

\section{Degradation of Hyaluronate by SDSE}

We first analyzed HA degradation by GAS-476, GBS-COH1, and SDSE-124 on hyaluronate-containing agar plates. As shown in Figure 2A, SDSE-124 exhibited high levels of HA degradation at comparatively low concentrations $\left(\mathrm{OD}_{600}=0.05\right.$ and 0.005$)$. We detected degraded $\mathrm{HA}$ on GBS-COH1 plates only at the high concentrations $\left(\mathrm{OD}_{600}=5\right.$ and 0.5$)$ and only partially degraded HA at the highest concentration of GAS-476 $\left(\mathrm{OD}_{600}=5\right)$. To determine whether the capacity to degrade HA varied depending on the specific isolate, several SDSE, GBS, and GAS strains were spotted at $\mathrm{OD}_{600}=0.5$ (Figure 2B). Significant HA degradation was observed on plates spotted with SDSE strains from patients with streptococcal toxic shock syndrome (strains C167 and TPCH-F36), as well as those isolated from joint fluid (strain KNZ01), blood (strain KNZ03), and open wounds (strains KNZ10 and KNZ16); these results indicated that all SDSE strains could degrade HA. By contrast, deletion of hylD gene $(\Delta h y l D)$ in SDSE-124 completely eliminated the capacity for HA degradation. Interestingly, although SDSE TPCH-F36 expresses a phage-related hyaluronate lyase gene encoding HylP (WP_003058192.1), its capacity to degrade HA was not significantly higher than that of the KNZ01, KNZ03, KNZ10, or KNZ16 strains; these results suggested that the expression or activity of HylD is more significant than that of HylP in this SDSE strain. Although GBS-COH1 could degrade $\mathrm{HA}$ at high concentrations, the area of $\mathrm{HA}$ degradation surrounding the GBS-COH1 spots was significantly smaller compared with those containing the SDSE strains. No HA degradation was observed surrounding the spots containing GBS-A909, GAS-476, or GAS-M1SMD at $\mathrm{OD}_{600}=0.5$.

We then proceeded to compare expression levels of the genes encoding hyl and $u g l$ (UGL, ENZYME entry EC 3.2.1.179). SDSE-124 and GBS-COH1 bacteria were cultured in hyaluronate- and/or glucose-containing medium without agar and collected for RT-qPCR analysis. As shown in Figure 2C, expression of both hyl and ugl was detected at dramatically higher levels in SDSE-124 compared with GBS-COH1. These results suggest that SDSE can express comparatively large amounts of both Hyl and UGL that facilitate rapid HA degradation. The high expression of hyl and ugl in SDE-124 was not observed in glucose-containing medium, indicating that HA acts as a signal to produce more HylD and UGL in SDSE.

\section{Growth in Chemically Defined Minimal Medium}

Next, we analyzed the growth rates of GAS, GBS, and SDSE strains in chemically defined minimal media (CDM). 
A

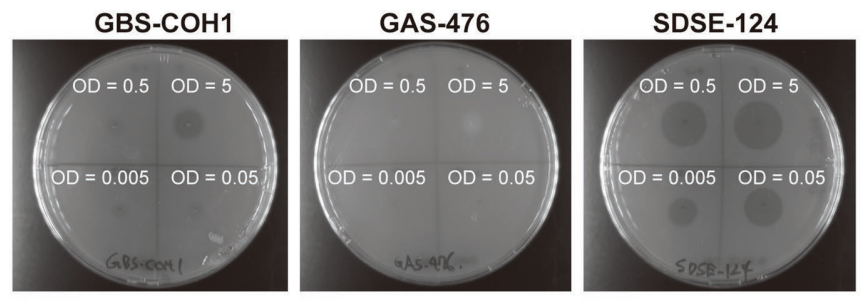

B

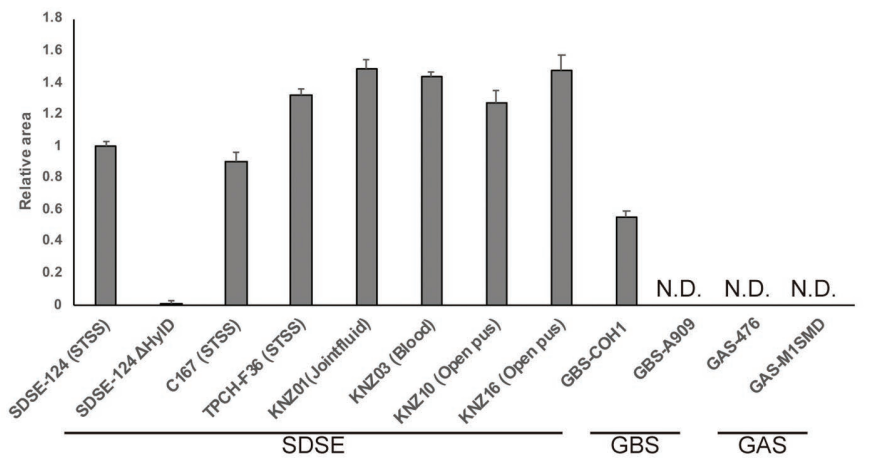

C
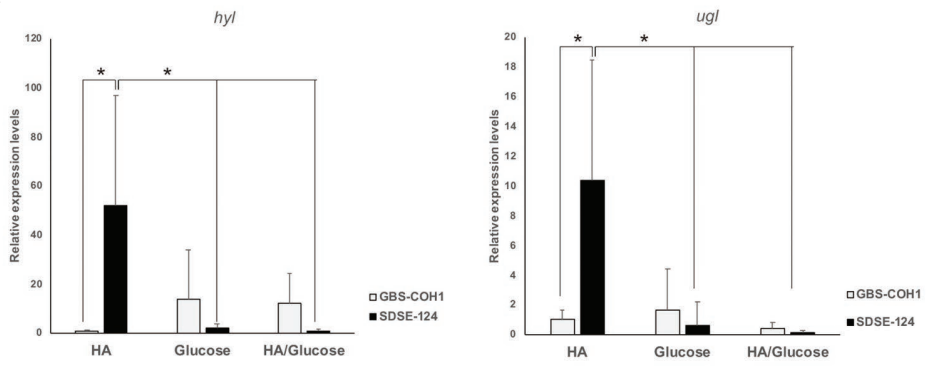

FIGURE 2 | Hyaluronate degradation. (A) Hyaluronate degradation on agar plates. GAS-476, GBS-COH1, and SDSE-124 were grown on brain-heart infusion (BHI) medium overnight and resuspended in fresh $\mathrm{BH}$ at the indicated $\mathrm{OD}_{600}$. One microliter of each bacterial solution was spotted on hyaluronate-containing agar plates. By soaking the plates with $2 \mathrm{~N}$ acetic acid, degraded hyaluronate was detected around the bacterial spots after overnight culture. (B) Relative area after spotting $1 \mu \mathrm{l}$ of $\mathrm{OD}_{600}=0.5$ solution followed by overnight culture. The areas were measured using Image $\mathrm{J}$ software. (C) Quantitative evaluation of hyl and ugl gene expression after $6 \mathrm{~h}$ of incubation in $0.4 \mathrm{mg} / \mathrm{ml}$ hyaluronate (HA)- and/or $10 \mathrm{mg} / \mathrm{ml}$ glucose-containing medium without agar. The average and standard deviation were calculated from three separate experiments performed in quadruplicate. Data were normalized to expression levels of the mutS gene. Relative expression levels were calculated as 1 using the averages of the hyl and ugl expression of GBS- $\mathrm{COH} 1$ in HA-containing medium. Values of $p$ were calculated using Student's $t$-test; ${ }^{*} p<0.01$.

None of the bacteria grew in the CDM in the absence of added carbohydrate (Figure 3A). In the presence of $10 \mathrm{mg} /$ $\mathrm{ml}$ glucose, GBS-COH1 entered its exponential phase after 4-6 $\mathrm{h}$ of incubation and expanded at a rate that exceeded that of SDSE-124 wild-type and SDSE-124 $\Delta$ hylD mutant bacteria (Figure 3B). SDSE-124 wild-type, but not $\Delta h y l D$ mutant, entered the exponential phase after 6-10 h of incubation and expanded at a rate that exceeded those of GBS-COH1 and GAS-476 in the presence of low $(0.4 \mathrm{mg} / \mathrm{ml})$ or high $(2.0 \mathrm{mg} / \mathrm{ml})$ concentrations of HA (Figures 3C,D). After a prolonged incubation $\left(96 \mathrm{~h}\right.$ ), the $\mathrm{OD}_{600}$ of GBS-COH1 and GAS-476 at the plateau phase were equal to or higher than that determined for SDSE-124 (Figures 3E,F). These results indicate that SDSE can grow and divide more rapidly using $\mathrm{HA}$ as a carbon source than the other $\beta$-hemolytic streptococci evaluated here. $\mathrm{OD}_{600}$ of SDSE decreased after $48-96 \mathrm{~h}$ of incubation, suggesting that SDSE grows rapidly but reaches a plateau sooner $(24 \mathrm{~h})$.

\section{Enzymatic Activity}

The enzymatic activity of HylD has not yet been characterized. To measure the enzymatic activities of the Hyl proteins in vitro, we overexpressed HylB and HylD in E. coli and purified them from bacterial lysates. As shown in Figure $\mathbf{4 A}$, the optima for both $\mathrm{HylB}$ and HylD was $\mathrm{pH} 6.5$; interestingly, the enzymatic activity of HylD at this $\mathrm{pH}$ was significantly higher than that of HylB, and the activity of HylD was twice that of $\mathrm{HylB}$ at $\mathrm{pH}$ 6.0. The enzymatic activity of HylB was slightly higher than that of HylD at $\mathrm{pH} 7.5$, which approximates the physiologic $\mathrm{pH}$ of human blood $(\mathrm{pH} 7.4)$. 

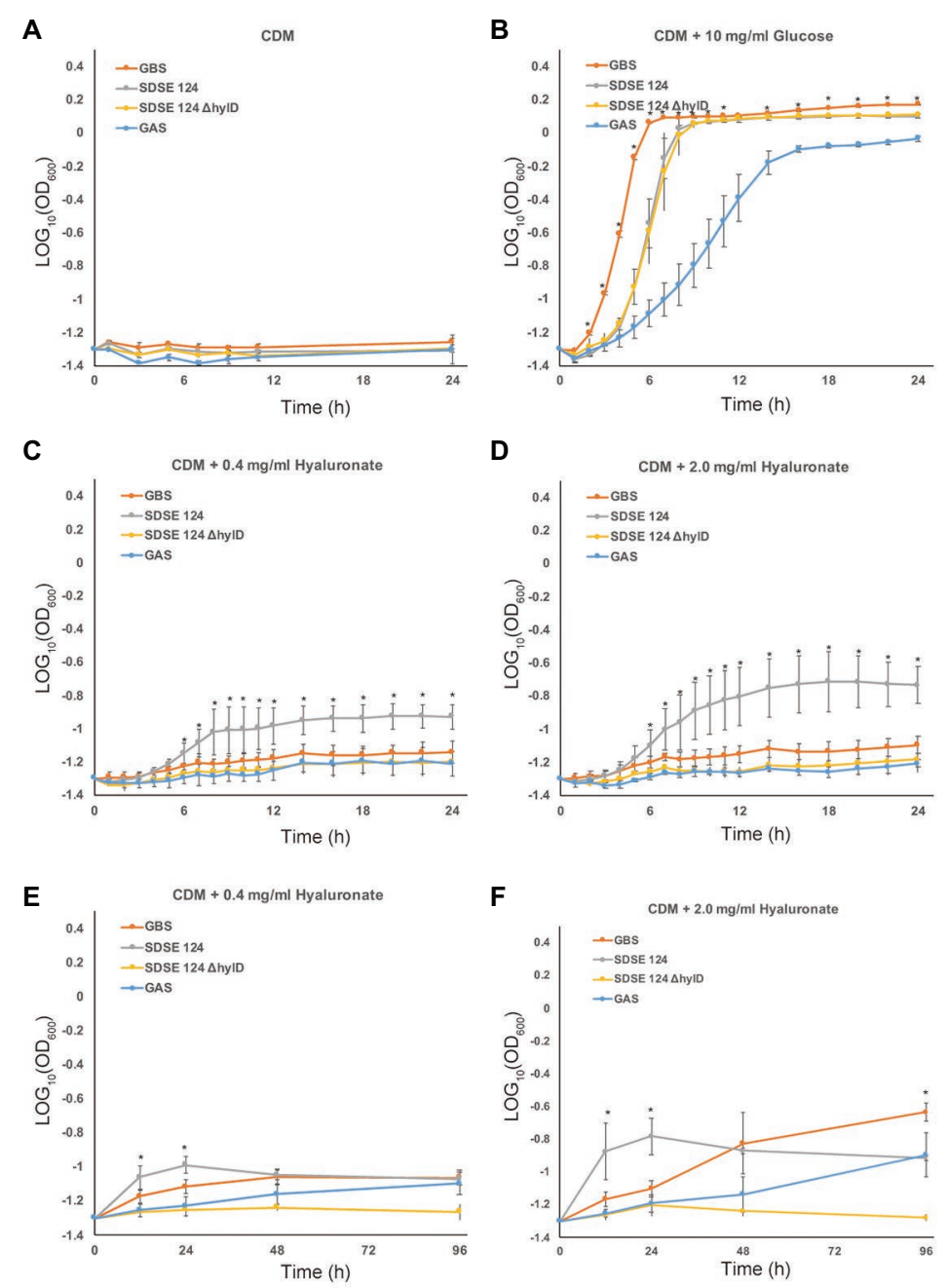

FIGURE 3 | Growth curves in minimal medium. Bacterial growth was assessed during a $24 \mathrm{~h}$ of incubation in chemically defined medium (CDM) containing (A) no carbohydrate, (B) $10 \mathrm{mg} / \mathrm{ml}$ glucose, (C) $0.4 \mathrm{mg} / \mathrm{ml} \mathrm{HA}$, or (D) $2.0 \mathrm{mg} / \mathrm{ml} \mathrm{HA}$. Bacterial growth was assessed during a $96 \mathrm{~h}$ incubation in CDM containing (E) $0.4 \mathrm{mg} / \mathrm{ml} \mathrm{HA}$ or $\mathbf{( F )} 2.0 \mathrm{mg} / \mathrm{ml} \mathrm{HA}$. Bacterial strains GAS-476, GBS-COH1, SDSE-124, and SDSE-124AhylD were cultivated in BHI medium overnight, collected by centrifugation, and resuspended in each medium as indicated. The average and standard deviation at each point were calculated from three separate experiments performed in triplicate. Analysis of variance and Bonferroni post hoc test were conducted to assess overall difference in $\mathrm{OD}_{600}$ at each time point; ${ }^{*} p<0.01$.

HylD was inactive at alkaline $\mathrm{pH}(\mathrm{pH} 8.5$ and 9.0), whereas HylB retained some of its activity. A surface skin $\mathrm{pH}$ of 6.0 has been reported for elderly individuals and patients diagnosed with diabetes mellitus. These data suggest that HylD may show significant HA-degrading activity on skin tissue at this pH. As shown in Figure 4B, UGL acted on Hyl-produced disaccharides but not on full-length HA.

\section{Expression of hylD and UGL in Mice}

As shown in Figure 2C, HA induced the expression of both $h y l$ and $u g l$ genes in SDSE in experiments conducted in vitro. We proceeded to analyze the expression of these two genes in bacteria used to infect mice. SDSE-124 was injected into the intraperitoneal cavity of mice and collected at the hours post-inoculation as indicated. We observed significant upregulation of hylD in SDSE-124 recovered from mice after $6 \mathrm{~h}$ (Figure 5A, left panel). As anticipated, no expression was detected in SDSE-124 $\Delta$ hylD recovered at this same time point. The gene encoding $u g l$ was also upregulated in SDSE-124 that were recovered at $6 \mathrm{~h}$; no upregulation was detected in recovered SDSE-124 4 hylD cells (Figure 5A, right panel); these results suggested that SDSE-124 coordinately regulates these genes so that HA is completely eliminated. The fact that we observed

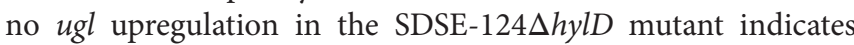
that either (a) disaccharides produced by HylD triggers $u g l$ expression or (b) $u g l$ expression is triggered by HylD itself.

\section{Lethal Infection With SDSE}

Infection with SDSE-124 and SDSE-124DhylD was compared in response to intraperitoneal injection of equivalent numbers 
of bacteria into C57BL6/J mice. We found that $53 \%$ of the mice inoculated with SDSE-124 (eight of 15 mice) died within 1 day post-infection; $67 \%$ mortality was observed at 7 days

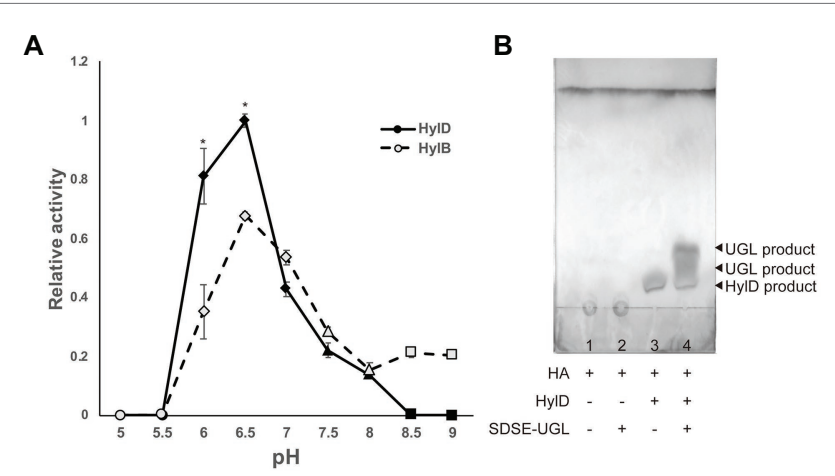

FIGURE 4 | Enzymatic activities of Hyl and degradation profiles for UGL. (A) $\mathrm{pH}$-dependent activities of Hyl proteins from GBS (HylB) and SDSE (HylD). Ten microliters of purified HylB and HyID $(0.05 \mathrm{mg} / \mathrm{ml}$ in $5 \mathrm{mM}$ HEPES- $\mathrm{NaOH}$, $\mathrm{pH}$ 7.0) was added to solutions of $500 \mu \mathrm{l}$ of $1 \mathrm{mg} / \mathrm{ml} \mathrm{HA}$ in $0.2 \mathrm{M}$ citrate (circle), MOPS-NaOH (rhomb), HEPES-NaOH (triangle), or glycine- $\mathrm{NaOH}$ (square). Relative activity was calculated as the increase in $\mathrm{OD}_{235}$ over time. $P$ values were calculated using Student's $t$-test; ${ }^{*} p<0.01$. (B) Product analysis using thin layer chromatography. The HA solution $(1 \mathrm{mg} / \mathrm{ml})$ was incubated with PBS (1), $5 \mu \mathrm{g} / \mathrm{ml}$ SDSE-UGL (2), $5 \mu \mathrm{g} / \mathrm{ml} \mathrm{HyID} \mathrm{(3),} \mathrm{or} \mathrm{both} 5 \mu \mathrm{g} /$ $\mathrm{ml} \mathrm{SDSE-UGL}$ and $5 \mu \mathrm{g} / \mathrm{ml} \mathrm{HyID} \mathrm{(4)} \mathrm{at} \mathrm{pH} 6.0$ (0.1 M citrate). The products were then spotted on a plate, separated with thin layer chromatography using a solvent system of 1-butanol/acetic acid/water (4:1:1, v/v), and visualized by spraying them with $5 \%$ sodium phosphomolybdate $n$-hydrate. post-inoculation (Figure 5B). By contrast, only $13 \%$ of the SDSE-124 $\Delta$ hylD-inoculated mice (two of 16 mice) died overall. Survival was significantly higher in response to infection with the SDSE-124DhylD strain; these results suggest that $\mathrm{HylD}$ is a critical element promoting the pathogenicity of SDSE.

\section{Skin Wound Infection With SDSE}

We determined whether HylD is involved in skin infection considering that SDSE is omnipresent on human skin. SDSE cannot invade normal mouse skin because it lacks the speB gene that encodes for the cysteine protease $\mathrm{SpeB}$, which is critically important for mouse skin invasion (Lukomski et al., 1999; Sumitomo et al., 2018). In this study, we tested a skin wound infection model with SDSE. Wounds were made with a biopsy punch, covered with dressing to maintain a moist environment for $24 \mathrm{~h}$, and infected with SDSE-124 or SDSE$124 \Delta h y l D$ for 2 days. Intensive infiltration of neutrophils was observed around the wound areas infected with SDSE-124 (Figure 6A), while fewer neutrophils were infiltrated around

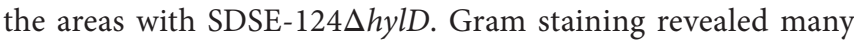

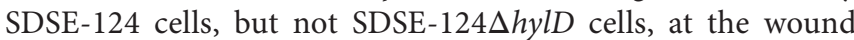
areas (Figure 6B). Consistent with neutrophil filtration, serum IL-6 levels were significantly higher in SDSE-124-infected

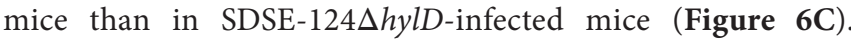
These results suggest that HylD is involved in the inflammation of wounded skin.

A

hyl

$u g l$
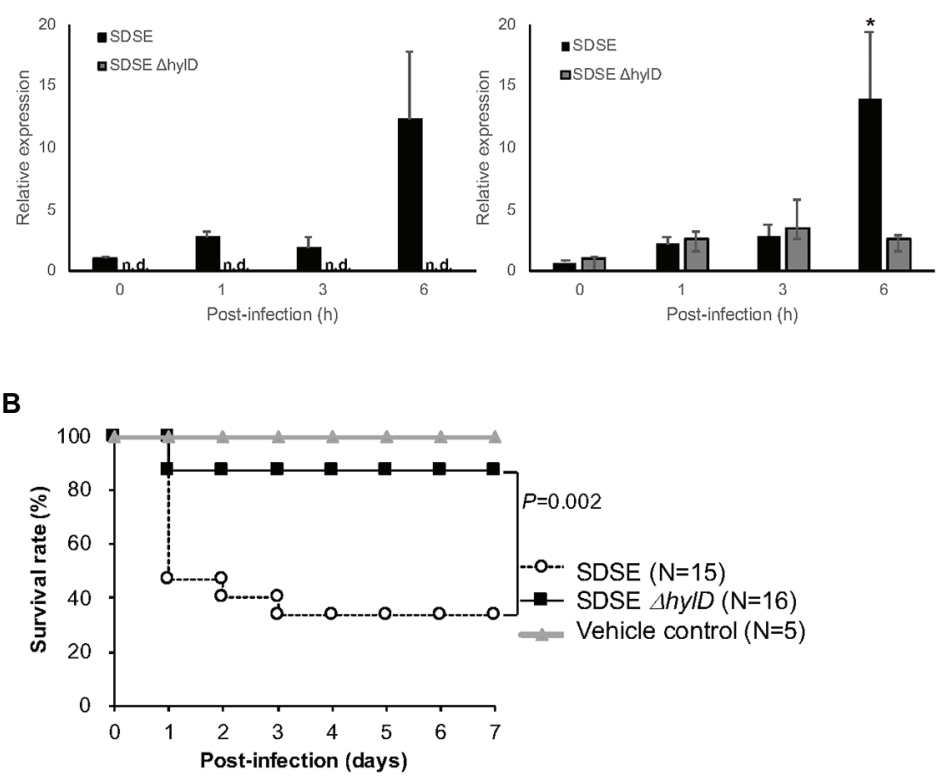

FIGURE 5 | Impact of hylD gene deletion on SDSE infection in mice. (A) The relative expression of hylD and ugl after intraperitoneal injection and recovery. SDSE-124 and SDSE-124 $\Delta$ hylD were injected intraperitoneally and collected at the indicated times; ${ }^{*} p<0.01$ according to Student's $t$-test. Relative expression

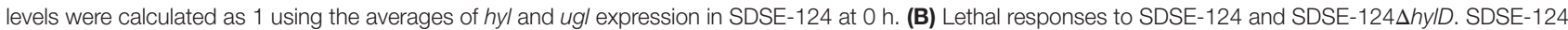
and SDSE-124AhyID were injected intraperitoneally at $2.0 \times 10^{6} \mathrm{CFU} /$ mouse, and survival was monitored for 7 days. The value of $p$ was calculated using the logrank test. BHI medium was injected for vehicle control. 
A

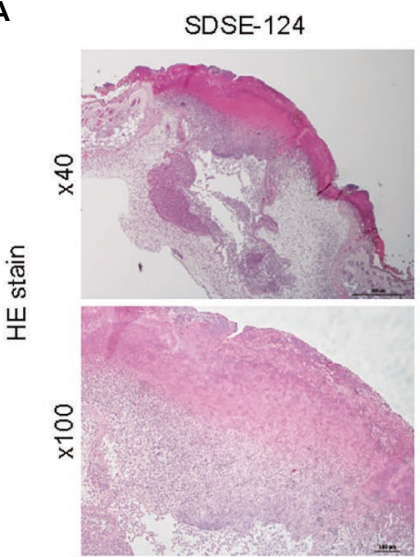

B

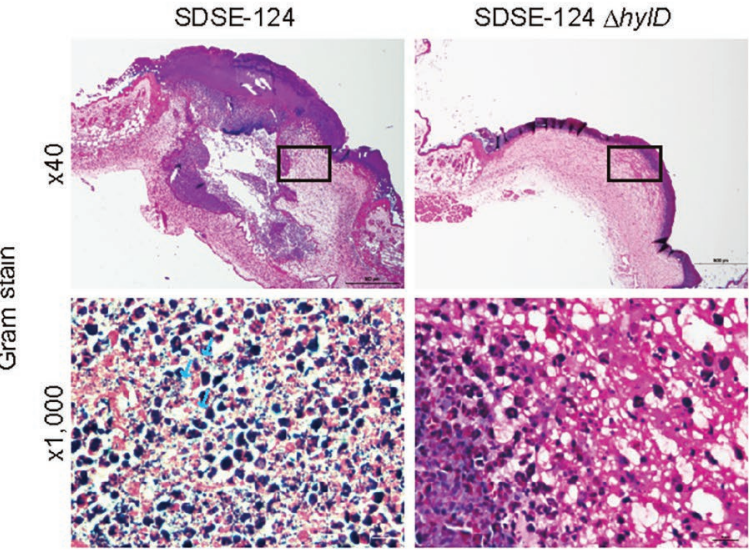

C

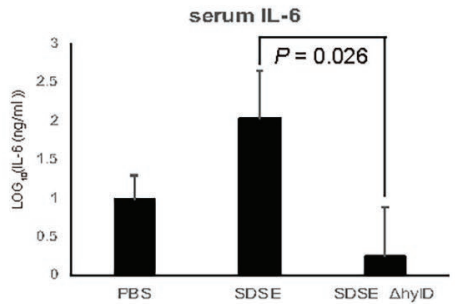

FIGURE 6 | Wound infection. (A) H\&E staining and (B) Gram staining of tissue sections taken from the wound areas. An arrow indicates the bacteria cells. (C) IL-6 concentrations of SDSE-infected mice. Data represent the mean \pm SD of log-transformation of IL-6 concentrations. Mice were infected with SDSE strains $\left(1.4 \times 10^{6} \mathrm{CFU} /\right.$ mouse) via the wound areas. On day 2 after infection, the mice were euthanized with Sevofrane, blood was immediately collected for IL-6 tests, and samples of the wound areas were taken for H\&E and Gram stains. The value of $p$ was calculated using Student's $t$-test.

\section{DISCUSSION}

Although SDSE is among the normal human flora, this bacterial species has also been associated with a significant diversity of diseases. Specifically, SDSE has been associated with the pathogenesis of pharyngitis, acute post-streptococcal glomerulonephritis, and acute rheumatic fever (Haidan et al., 2000; Bassett et al., 2009). While GAS and GBS possess a cluster of three genes (hasA, hasB, and hasC) related to HA synthesis, SDSE lacks has $A$ and has $B$ genes, resulting in absence of HA capsules. Although the clinical features of SDSE-associated diseases closely resemble GAS infections, the patient characteristics differ substantially (Watanabe et al., 2016). Notably, SDSE is a major cause of invasive $\beta$-streptococcal infections among the elderly in Japan (Takahashi et al., 2010). Most patients who present with SDSE bacteremia have other co-morbidities such as malignant tumors and diabetes mellitus (Broyles et al., 2009; Rantala, 2014). We previously explored physiologic responses to these bacteria and found that SDSE is particularly lethal in diabetic mice (Ogura et al., 2018). In this study, we focused on the relationship between HA degradation and pathogenic disease associated with SDSE. HA concentrations vary significantly among biological fluids and tissues (Cowman et al., 2015). The human skin contains approximately $0.4 \mathrm{mg} / \mathrm{g} \mathrm{HA}$, primarily found in the dermis (Tammi et al., 1994). HA concentration within the human knee joint is higher $(2-3 \mathrm{mg} / \mathrm{ml})$ than in any other fluid or tissue in the human body (Balazs et al., 1967; Dahl et al., 1985). SDSE is often isolated from the skins and joint fluids (Wajima et al., 2016; Matsue et al., 2020).

Streptococcus dysgalactiae subsp. equisimilis hyaluronate lyase (HylD) is homologous to GBS (HylB) at a $44 \%$ amino acid sequence identity. Because of this homology, the virulence factor database recognizes the SDSE $h y l D$ gene as analogous to the GBS hylB gene (Chen et al., 2004). Homology modeling indicated that surface charge characteristic of the modeled HylD protein resembles that of HylB despite significant differences in computed pIs (5.5 and 8.8 for HylD and HylB, respectively, Figure 1C). However, the two enzymes differ with respect to enzymatic activity (Figure $4 \mathbf{A}$ ). In human skin, $\mathrm{HA}$ is localized not only in cellular matrix whose $\mathrm{pH}$ is neutral but also surface skin such as stratum corneum whose $\mathrm{pH}$ is acidic (Sakai et al., 2000). Neither enzyme can digest HA at $\mathrm{pH} 5.0$ nor at $\mathrm{pH} 5.5$, which is the typical $\mathrm{pH}$ for the surface skin of younger individuals. HylD was twice as active as HylB at $\mathrm{pH}$ 6.0, which is the typical $\mathrm{pH}$ for an aged surface skin. This increase in $\mathrm{pH}$ may relate to reduced expression of the sodium-hydrogen exchanger (NHE1) and filaggrin associated with reductions in acidifying free fatty acids, sebum, and sweat (Choi et al., 2007; Levin and Maibach, 2008). Surface skin $\mathrm{pH}$ is also increased among those diagnosed with diabetes mellitus (Behm et al., 2012). Our data suggest that HylD could degrade HA on the skins of elderly individuals and those who diagnosed with diabetes mellitus are highly susceptible to SDSE infection.

As shown in Supplementary Figure S3, hylB and hylD are designated in the genomes differently among GBS and SDSE. We determined that SDSE can degrade HA more effectively than do equal amounts of GBS and GAS; this observation relates to augmented production and secretion of HylD and UGL (Figure 2). We also found that SDSE grew rapidly in chemically defined minimal media containing $\mathrm{HA}$ as the sole carbohydrate source (Figure 3). Moreover, expression of hylD gene was significantly upregulated in SDSE injected intraperitoneally in mice (Figure 5A). HylD degrades HA via 
a $\beta$-elimination reaction to unsaturated disaccharides, which are then hydrolyzed by UGL. As shown in Figure $\mathbf{5 A}$, SDSE-124 4 hylD did not upregulate the $u g l$ gene, suggesting the accumulation of unsaturated disaccharides may trigger its expression; a further understanding of this mechanism might be pursued in future studies. Of note, Kolar et al. reported that unsaturated disaccharides generated in GBS block inflammatory responses and permit the bacterial cells to escape immune surveillance (Kolar et al., 2015). Following this line of thinking, high concentrations of UGL may lead to a decrease in the concentration of accumulated disaccharides and thus promote inflammatory responses; this will represent a significant disadvantage from the perspective of bacterial evasion.

It remains unclear as to whether disaccharides generated by the actions of HylD can limit inflammation and promote SDSE-124 immune evasion.

We found that deletion of hylD gene resulted in a significant decrease in mortality among infected C57BL6/J mice (Figure 5B). Moreover, in open wounds, SDSE wild-type but not $\Delta$ hylD mutant resided on day 2 post-infection, resulting in infiltration of neutrophils and increase of serum IL-6 level (Figure 6). These results suggest that HylD is involved in the pathogenicity of SDSE through nutrient degradation on host skins. By contrast, deletion of the hylB gene in GBS resulted in amplified lethality that was eliminated in infection studies conducted in Tlr2 gene knockout mice (Kolar et al., 2015). Of note, GBS but not SDSE secrete hyaluronate capsules that promote immune evasion; deletion of the $h y l B$ gene in GBS may promote formation of a more substantial capsule and thereby promote lethal infection.

It remains unclear how SDSE typically found on the skin invades and enters systemic circulation to generate life-threatening bacteremia. SDSE does not produce cysteine protease SpeB, which is a critical element that promotes GAS invasion (Lukomski et al., 1999; Sumitomo et al., 2018). It is possible that SDSE does not transit through normal skin but can invade via sites of injury sites. Frenkel (2014) reported that HA is an integral component at the site of skin injury and promotes wound healing, indicating that SDSE can target HA accumulated at the healing sites. Further analysis will be required to elucidate the mechanism of SDSE invasion.

In conclusion, we characterized the expression of genes encoding for HylD and UGL in bacterial strain SDSE-124. These two enzymes were upregulated in response to HA. This investigation suggested that HylD and UGL play important roles in nutrient acquisition from hosts, followed by the bacterial pathogenicity damaging host tissues. This is the first report that has identified and characterized a specific virulence factor from SDSE, to the best of our knowledge.

\section{REFERENCES}

Balazs, E. A., Watson, D., Duff, I. F., and Roseman, S. (1967). Hyaluronic acid in synovial fluid. I. Molecular parameters of hyaluronic acid in normal and arthritic human fluids. Arthritis Rheum. 10, 357-376. doi: 10.1002/art.1780100407

Bassett, D. C. J., Read, S. E., Reid, H. F. M., Zabriskie, J. B., and Poon-King, T. (2009). Group G streptococci in healthy school-children and in patients

\section{DATA AVAILABILITY STATEMENT}

The original contributions presented in the study are included in the article/Supplementary Material, further inquiries can be directed to the corresponding author.

\section{ETHICS STATEMENT}

The animal study was reviewed and approved by the Committee for Genetic Recombination Experiments (Kanazawa University).

\section{AUTHOR CONTRIBUTIONS}

VAN performed the experiments. KO conceived this project, directed the study, and conducted enzymatic analysis. VAN and $\mathrm{KO}$ wrote the manuscript. MM contributed to mice experiments and improved the quality of the manuscript. NT contributed to study design for revision of the manuscript. KM contributed to design of wound infection experiments. YN contributed to sample preparation for histological analysis. TLH, YI, NS, and TW contributed to histological analysis of skin tissues. WH critically revised the manuscript and contributed to hyaluronate degradation assay and TLC analysis. SO contributed to ethical approval of the animal experiments and progress of the study. HI supervised VAN and directed the study. All authors contributed to the article and approved the submitted version.

\section{FUNDING}

This work was partly supported by JSPS Grant-in-Aid for Scientific Research C (number 18K07133 to KO) and Takeda Science Foundation (KO).

\section{ACKNOWLEDGMENTS}

We thank Advanced Science Research Center (Kanazawa University) for Sanger sequencing and Enago (www.enago.jp) for the English language review.

\section{SUPPLEMENTARY MATERIAL}

The Supplementary Material for this article can be found online at: https://www.frontiersin.org/articles/10.3389/fmicb.2020.552418/ full\#supplementary-material

with glomerulonephritis in Trinidad. J. Hyg. 94, 61-68. doi: 10.1017/ s0022172400061131

Baumann, L. (2007). Skin ageing and its treatment. J. Pathol. 211, 241-251. doi: 10.1002/path.2098

Behm, B., Schreml, S., Landthaler, M., and Babilas, P. (2012). Skin signs in diabetes mellitus. J. Eur. Acad. Dermatol. Venereol. 26, 1203-1211. doi: 10.1111/j.1468-3083.2012.04475.x 
Bjellqvist, B., Hughes, G. J., Pasquali, C., Paquet, N., Ravier, F., Sanchez, J. C., et al. (1993). The focusing positions of polypeptides in immobilized $\mathrm{pH}$ gradients can be predicted from their amino acid sequences. Electrophoresis 14, 1023-1031. doi: 10.1002/elps.11501401163

Brandt, C. M. M., and Spellerberg, B. (2009). Human infections due to Streptococcus dysgalactiae subspecies equisimilis. Clin. Infect. Dis. 49, 766-772. doi: 10.1086/605085

Broyles, L. N., Van Beneden, C., Beall, B., Facklam, R., Shewmaker, P. L., Malpiedi, P., et al. (2009). Population-based study of invasive disease due to beta-hemolytic streptococci of groups other than A and B. Clin. Infect. Dis. 48, 706-712. doi: 10.1086/597035

Chen, L., Yang, J., Yu, J., Yao, Z., Sun, L., Shen, Y., et al. (2004). VFDB: a reference database for bacterial virulence factors. Nucleic Acids Res. 33, D325-D328. doi: 10.1093/nar/gki008

Choi, E. H., Man, M. Q., Xu, P., Xin, S., Liu, Z., Crumrine, D. A., et al. (2007). Stratum corneum acidification is impaired in moderately aged human and murine skin. J. Invest. Dermatol. 127, 2847-2856. doi: 10.1038/sj.jid.5700913

Cowman, M. K., Lee, H. G., Schwertfeger, K. L., McCarthy, J. B., and Turley, E. A. (2015). The content and size of hyaluronan in biological fluids and tissues. Front. Immunol. 6:261. doi: 10.3389/fimmu.2015.00261

Dahl, L. B., Dahl, I. M. S., Engstrom-Laurent, A., and Granath, K. (1985). Concentration and molecular weight of sodium hyaluronate in synovial fluid from patients with rheumatoid arthritis and other arthropathies. Ann. Rheum. Dis. 44, 817-822. doi: 10.1136/ard.44.12.817

Diehl, K. H., Hull, R., Morton, D., Pfister, R., Rabemampianina, Y., Smith, D., et al. (2001). A good practice guide to the administration of substances and removal of blood, including routes and volumes. J. Appl. Toxicol. 21, 15-23. doi: 10.1002/jat.727

Eswar, N., Webb, B., Marti-Renom, M. A., Madhusudhan, M. S., Eramian, D., Shen, M., et al. (2006). Comparative protein structure modeling using modeller. Curr. Protoc. Bioinformatics 15, 5.6.1-5.6.30. doi: 10.1002/0471250953. bi0506s 15

Falaleeva, M., Zurek, O. W., Watkins, R. L., Reed, R. W., Ali, H., Sumby, P., et al. (2014). Transcription of the Streptococcus pyogenes hyaluronic acid capsule biosynthesis operon is regulated by previously unknown upstream elements. Infect. Immun. 82, 5293-5307. doi: 10.1128/IAI.02035-14

Frenkel, J. S. (2014). The role of hyaluronan in wound healing. Int. Wound J. 11, 159-163. doi: 10.1111/j.1742-481X.2012.01057.x

Haidan, A., Talay, S. R., Rohde, M., Sriprakash, K. S., Currie, B. J., and Chhatwal, G. S. (2000). Pharyngeal carriage of group C and group G streptococci and acute rheumatic fever in an aboriginal population. Lancet 356, 1167-1169. doi: 10.1016/S0140-6736(00)02765-3

Hashimoto, W., Kobayashi, E., Nankai, H., Sato, N., Miya, T., Kawai, S., et al. (1999). Unsaturated glucuronyl hydrolase of Bacillus sp. GL1: novel enzyme prerequisite for metabolism of unsaturated oligosaccharides produced by polysaccharide lyases. Arch. Biochem. Biophys. 368, 367-374. doi: 10.1006/ abbi.1999.1305

Heath, A., DiRita, V. J., Barg, N. L., and Engleberg, N. C. (1999). A twocomponent regulatory system, CsrR-CsrS, represses expression of three Streptococcus pyogenes virulence factors, hyaluronic acid capsule, streptolysin S, and pyrogenic exotoxin B. Infect. Immun. 67, 5298-5305. doi: 10.1128/ IAI.67.10.5298-5305.1999

Hynes, W. (2004). Virulence factors of the group A streptococci and genes that regulate their expression. Front. Biosci. 9:1491. doi: 10.2741/1491

Ishihara, H., Ogura, K., Miyoshi-Akiyama, T., Nakamura, M., Kaya, H., and Okamoto, S. (2020). Prevalence and genomic characterization of group A Streptococcus dysgalactiae subsp. equisimilis isolated from patients with invasive infections in Toyama prefecture, Japan. Microbiol. Immunol. 64, 113-122. doi: $10.1111 / 1348-0421.12760$

Jedrzejas, M. J., and Chantalat, L. (2000). Structural studies of Streptococcus agalactiae hyaluronate lyase. Acta Crystallogr. D Struct. Biol. 56, 460-463. doi: 10.1107/S0907444900000706

Juhlin, L. (1997). Hyaluronan in skin. J. Intern. Med. 242, 61-66. doi: 10.1046/j. 1365-2796.1997.00175.x

Kolar, S. L., Kyme, P., Tseng, C. W., Soliman, A., Kaplan, A., Liang, J., et al. (2015). Group B Streptococcus evades host immunity by degrading hyaluronan. Cell Host Microbe 18, 694-704. doi: 10.1016/J.CHOM.2015.11.001

Levin, J., and Maibach, H. (2008). Human skin buffering capacity: an overview. Skin Res. Technol. 14, 121-126. doi: 10.1111/j.1600-0846.2007.00271.x
Lukomski, S., Montgomery, C. A., Rurangirwa, J., Geske, R. S., Barrish, J. P., Adams, G. J., et al. (1999). Extracellular cysteine protease produced by Streptococcus pyogenes participates in the pathogenesis of invasive skin infection and dissemination in mice. Infect. Immun. 67, 1779-1788.

Maruyama, Y., Nakamichi, Y., Itoh, T., Mikami, B., Hashimoto, W., and Murata, K. (2009). Substrate specificity of streptococcal unsaturated glucuronyl hydrolases for sulfated glycosaminoglycan. J. Biol. Chem. 284, 18059-18069. doi: 10.1074/ jbc.M109.005660

Matsue, M., Ogura, K., Sugiyama, H., Miyoshi-Akiyama, T., Takemori-Sakai, Y., Iwata, Y., et al. (2020). Pathogenicity characterization of prevalent-type Streptococcus dysgalactiae subsp. equisimilis strains. Front. Microbiol. 11:97. doi: $10.3389 /$ fmicb. 2020.00097

Meyer, L. J. M., and Stern, R. (1994). Age-dependent changes of hyaluronan in human skin. J. Invest. Dermatol. 102, 385-389. doi: 10.1111/1523-1747. EP12371800

Mukai, K., Urai, T., Asano, K., Nakajima, Y., and Nakatani, T. (2016). Evaluation of effects of topical estradiol benzoate application on cutaneous wound healing in ovariectomized female mice. PLoS One 11:e0163560. doi: 10.1371/ journal.pone. 0163560

Ogura, K., Okumura, K., Shimizu, Y., Kirikae, T., and Miyoshi-Akiyama, T. (2018). Pathogenicity induced by invasive infection of Streptococcus dysgalactiae subsp. equisimilis in a mouse model of diabetes. Front. Microbiol. 9:2128. doi: $10.3389 /$ fmicb.2018.02128

Oiki, S., Nakamichi, Y., Maruyama, Y., Mikami, B., Murata, K., and Hashimoto, W. (2019). Streptococcal phosphotransferase system imports unsaturated hyaluronan disaccharide derived from host extracellular matrices. PLoS One 14:e0224753. doi: 10.1371/journal.pone.0224753

Pettersen, E. F., Goddard, T. D., Huang, C. C., Couch, G. S., Greenblatt, D. M., Meng, E. C., et al. (2004). UCSF chimera - a visualization system for exploratory research and analysis. J. Comput. Chem. 25, 1605-1612. doi: $10.1002 /$ jcc. 20084

Rantala, S. (2014). Streptococcus dysgalactiae subsp. equisimilis bacteremia: an emerging infection. Eur. J. Clin. Microbiol. Infect. Dis. 33, 1303-1310. doi: 10.1007/s10096-014-2092-0

Reed, R. K., Lilja, K., and Laurent, T. C. (1988). Hyaluronan in the rat with special reference to the skin. Acta Physiol. Scand. 134, 405-411. doi: 10.1111/ j.1748-1716.1988.tb08508.x

Sakai, S., Yasuda, R., Sayo, T., Ishikawa, O., and Inoue, S. (2000). Hyaluronan exists in the normal stratum corneum. J. Invest. Dermatol. 114, 1184-1187. doi: 10.1046/j.1523-1747.2000.00992.x

Shimomura, Y., Okumura, K., Murayama, S. Y., Yagi, J., Ubukata, K., Kirikae, T., et al. (2011). Complete genome sequencing and analysis of a Lancefield group G Streptococcus dysgalactiae subsp. equisimilis strain causing streptococcal toxic shock syndrome (STSS). BMC Genomics 12:17. doi: 10.1186/14712164-12-17

Smith, R. F., and Willett, N. P. (1968). Rapid plate method for screening hyaluronidase and chondroitin sulfatase-producing microorganisms. Appl. Microbiol. 16, 1434-1436. doi: 10.1128/aem.16.9.1434-1436.1968

Stollerman, G. H., and Dale, J. B. (2008). The importance of the group A streptococcus capsule in the pathogenesis of human infections: a historical perspective. Clin. Infect. Dis. 46, 1038-1045. doi: 10.1086/529194

Sumitomo, T., Mori, Y., Nakamura, Y., Honda-Ogawa, M., Nakagawa, S., Yamaguchi, M., et al. (2018). Streptococcal cysteine protease-mediated cleavage of desmogleins is involved in the pathogenesis of cutaneous infection. Front. Cell. Infect. Microbiol. 8:10. doi: 10.3389/fcimb.2018.00010

Takahashi, T., Sunaoshi, K., Sunakawa, K., Fujishima, S., Watanabe, H., Ubukata, K., et al. (2010). Clinical aspects of invasive infections with Streptococcus dysgalactiae ssp. equisimilis in Japan: differences with respect to Streptococcus pyogenes and Streptococcus agalactiae infections. Clin. Microbiol. Infect. 16, 1097-1103. doi: 10.1111/j.1469-0691.2009.03047.x

Takahashi, T., Ubukata, K., and Watanabe, H. (2011). Invasive infection caused by Streptococcus dysgalactiae subsp. equisimilis: characteristics of strains and clinical features. J. Infect. Chemother. 17, 1-10. doi: 10.1007/s10156-010-0084-2

Takamatsu, D., Osaki, M., and Sekizaki, T. (2001). Thermosensitive suicide vectors for gene replacement in Streptococcus suis. Plasmid 46, 140-148. doi: $10.1006 /$ plas.2001.1532

Tammi, R., Ågren, U. M., Tuhkanen, A. L., and Tammi, M. (1994). Hyaluronan metabolism in skin. Prog. Histochem. Cytochem. 29, 3-7. doi: 10.1016/ S0079-6336(11)80023-9 
Tamura, K., Peterson, D., Peterson, N., Stecher, G., Nei, M., and Kumar, S. (2011). MEGA5: molecular evolutionary genetics analysis using maximum likelihood, evolutionary distance, and maximum parsimony methods. Mol. Biol. Evol. 28, 2731-2739. doi: 10.1093/molbev/msr121

van de Rijn, I., and Kessler, R. E. (1980). Growth characteristics of group A streptococci in a new chemically defined medium. Infect. Immun. 27, 444-448. doi: 10.1128/iai.27.2.444-448.1980

Wajima, T., Morozumi, M., Hanada, S., Sunaoshi, K., Chiba, N., Iwata, S., et al. (2016). Molecular characterization of invasive Streptococcus dysgalactiae subsp. equisimilis, Japan. Emerg. Infect. Dis. 22, 247-254. doi: 10.3201/ eid 2202.141732

Watanabe, S., Shimomura, Y., Ubukata, K., Kirikae, T., and Miyoshi-Akiyama, T. (2013). Concomitant regulation of host tissue-destroying virulence factors and carbohydrate metabolism during invasive diseases induced by group $\mathrm{g}$ streptococci. J. Infect. Dis. 208, 1482-1493. doi: 10.1093/infdis/jit353

Watanabe, S., Takemoto, N., Ogura, K., and Miyoshi-Akiyama, T. (2016). Severe invasive streptococcal infection by Streptococcus pyogenes and Streptococcus dysgalactiae subsp. equisimilis. Microbiol. Immunol. 60, 1-9. doi: 10.1111/ 1348-0421.12334

Yung, L., Rashid, M., and Bräu, N. (2019). Group C Streptococcus dysgalactiae subsp. equisimilis (SDSE) endocarditis with endogenous endophthalmitis and aortic root abscess. IDCases 15:e0513. doi: 10.1016/j.idcr.2019.e00513

Conflict of Interest: The authors declare that the research was conducted in the absence of any commercial or financial relationships that could be construed as a potential conflict of interest.

Copyright $\odot 2020$ Nguyen, Ogura, Matsue, Takemoto, Mukai, Nakajima, Hoang, Iwata Sakai, Wada, Hashimoto, Okamoto and Ichimura. This is an open-access article distributed under the terms of the Creative Commons Attribution License (CC BY). The use, distribution or reproduction in other forums is permitted, provided the original author(s) and the copyright owner(s) are credited and that the original publication in this journal is cited, in accordance with accepted academic practice. No use, distribution or reproduction is permitted which does not comply with these terms. 